\title{
Quantifying turbulent wall shear stress in a subject specific human aorta using large eddy simulation
}

Jonas Lantz, Roland Gårdhagen and Matts Karlsson

\section{Linköping University Post Print}

N.B.: When citing this work, cite the original article.

Original Publication:

Jonas Lantz, Roland Gårdhagen and Matts Karlsson, Quantifying turbulent wall shear stress in a subject specific human aorta using large eddy simulation, 2012, Medical Engineering and Physics, (34), 8, 1139-1148.

http://dx.doi.org/10.1016/j.medengphy.2011.12.002

Copyright: Elsevier http://www.elsevier.com/

Postprint available at: Linköping University Electronic Press http://urn.kb.se/resolve?urn=urn:nbn:se:liu:diva-84887 


\title{
Quantifying Turbulent Wall Shear Stress in a Subject Specific Human Aorta Using Large Eddy Simulation
}

\author{
Jonas Lantz * \\ Department of Management \\ and Engineering, \\ Linköping University, \\ SE-581 83 Linköping, Sweden \\ phone: +46 13 282335; \\ fax: +46 13281101 ; \\ e-mail:jonas.lantz@liu.se
}

\author{
Roland Gårdhagen \\ Department of Management \\ and Engineering \\ Linköping University, \\ SE-581 83 Linköping, Sweden \\ phone: +46 13 281701; \\ fax: +46 13281101 ; \\ e-mail:roland.gardhagen@liu.se
}

\author{
Matts Karlsson \\ Department of Management \\ and Engineering \\ Linköping University, \\ SE-581 83 Linköping, Sweden \\ phone: +46 13281199 ; \\ fax: +46 13281101 ; \\ e-mail:matts.karlsson@liu.se
}

\begin{abstract}
In this study, large-eddy simulation (LES) is employed to calculate the disturbed flow field and the wall shear stress (WSS) in a subject specific human aorta. Velocity and geometry measurements using magnetic resonance imaging (MRI) are taken as input to the model to provide accurate boundary conditions and to assure the physiological relevance. In total, 50 consecutive cardiac cycles were simulated from which a phase average was computed to get a statistically reliable result. A decomposition similar to Reynolds decomposition is introduced, where the WSS signal is divided into a pulsating part (due to the mass flow rate) and a fluctuating part (originating from the disturbed flow). Oscillatory shear index (OSI) is plotted against time-averaged


WSS in a novel way and, locations on the aortic wall where elevated values existed could easily be found. In general, high and oscillating WSS values were found in the vicinity of the branches in the aortic arch, while low and oscillating WSS were present in the inner curvature of the descending aorta. The decomposition of WSS into a pulsating and a fluctuating part increases the understanding of how WSS affects the aortic wall, which enables both qualitative and quantitative comparisons.

Keywords: human aorta, atherosclerosis, wall shear stress, computational fluid dynamics, scale resolving turbulence model, Reynolds decomposition

\section{Introduction}

Vascular diseases have been correlated to locations with highly disturbed flow, predominantly around the aortic arch branches, and the bend of the descending aorta. In these locations the wall shear stress (WSS) has been found to be oscillating in both direction and magnitude, which might be a part of the genesis of vascular diseases [1-3]. Normal aortic flow is already a very complicated phenomenon, even without the presence of vascular disease. Blood is ejected from the heart into a complex geometry, which is characterized by curving, tapering, and branching.

Simulation of cardiovascular flow presents a number of challenges, which to be overcome require a combination of state of the art tools from engineering and medical disciplines. Numerous studies and advancement of technology during the last decades have now opened for detailed flow simulations, resolving most of the dynamics of the flow in realistic subject specific vessel models and hence allowing for a thorough quantification of the flow and related parameters.

Another path has been the development of the numerical methodology, particularly how to handle possible turbulence, including its onset, which is an area of intense research in itself. A series of numerical studies related to turbulence in the arterial system have been presented. Many of those compared 
numerical predictions to a series of measurements of turbulent flow in circular pipes with various degrees of symmetric stenoses for steady and unsteady flows [4-6]. The experiments demonstrated that steady flow turbulence can be expected even for Reynolds numbers of about 1000, if the flow is subject to any disturbance, e.g. an occlusion. In subsequent numerical studies various solvers and turbulence models have been evaluated and compared to the measurements; early works predominantly employed the Reynolds Averaged Navier-Stokes (RANS) equations, which provide a time averaged solution of the flow and some estimates of the fluctuating behavior. RANS models thus provide a somewhat limited representation of the flow lacking all dynamics of the turbulence, but nevertheless successful from an engineering perspective in that they might predict the mean properties of the flow and their interaction with the surrounding vessel in terms of the WSS. In addition they are cheap to run concerning the required computational power. For long, basic RANS models like the $k-\varepsilon$ and the $k-\omega$ fail to predict the transitional flow in the stenosed pipe [7-9]; a certain turbulence model is usually developed for a relatively limited number of applications, for which it provides reasonable results, whereas its potential is limited for other types of flows. A few studies have shown promising results of turbulence models, e.g. [10] in which the transitional variant of Menter's hybrid $k-\varepsilon / k-\omega$ SST model and the transitional scale adaptive simulation (SAS) model agreed reasonably with measurements done in [5].

To capture the dynamics of the turbulence, a scale resolving technique is required nonetheless. Direct numerical simulation (DNS) resolves all spatial and temporal scales of the flow, but is immensely expensive in terms of computational cost. In addition, the use of complex geometries is strongly limited due to the high order numerical schemes. Large eddy simulation (LES) on the other hand, resolves the larger turbulent eddies whereas the smaller ones are modeled. The computational cost compared to a DNS is significantly reduced, while most of the dynamics is captured in the calculations. So far, LES has mainly been obtained to flows in idealized geometries. Varghese et al. [11] studied the flow in the circular stenosed pipe, both the original axisymmetric and also a case with an eccentric stenosis, 
however only instantaneous results were reported. Recently, Tan et al. [12] presented a study in which the sub grid scale modeling was investigated in the same geometries as were considered by [11]. Statistical data was collected under a relatively short time, but indicated that the dynamic Smagorinsky model is capable to predict the flow and its dynamics through the axisymmetric stenosis, whereas the classic Smagorinsky was closer to DNS data [13] regarding the flow through an eccentric stenosis.

As the flow departs from being purely laminar, the time signal of a property will show a fluctuating behavior. In case of a non-pulsating flow, the fluctuations will exist around a constant mean value, while for a pulsating flow the fluctuations will be superimposed on a large time scale periodic signal. In a previous work we investigated WSS in a non-pulsating turbulent flow using the axisymmetric stenosed pipe [14]. Similar to the Reynolds decomposition, WSS was divided into a mean and a fluctuating component. It was found that recirculation zones were characterized by high time averaged WSS magnitude as well as significant fluctuations both in the axial and circumferential direction.

To take the previous work one step further, a natural step is to conduct scale resolving simulations of the flow in a subject specific model of a human aorta in order to characterize the flow and related hemodynamic parameters, particularly WSS. Our model contains anatomical data and velocity profiles acquired using MRI and use an advanced LES model to handle the flow field. Multiple cardiac cycles are considered to achieve statistical reliable results.

\section{Method}

\subsection{MRI Acquisition and Segmentation}

MRI acquisition was performed on a young healthy male, who gave informed consent to participate in the study. Aortic geometry and blood flow were acquired using a 1.5 T Philips Achieva MRI scanner and was obtained within a breath hold while time-resolved aortic flow rates were obtained by performing throughplane 2D velocity MRI acquisition perpendicular to the flow direction in the ascending 
and descending aorta. The $3 \mathrm{D}$ volume data was reconstructed to a resolution of $0.78 \times 0.78 \times 1.00 \mathrm{~mm}^{3}$ and segmented with the cardiac image analysis software package Segment [15], which gave a STLrepresentation of the inner aortic wall. The velocity measurements were reconstructed to 40 time-frames per cardiac cycle with a spatial resolution of $1.37 \times 1.37 \mathrm{~mm}^{2}$ which severed as an input to the simulation model, increasing the physiological accuracy.

\subsection{Computational Mesh}

The segmented geometry obtained from the MRI measurement was cut in the ascending aorta and in the thoracic aorta to provide well defined in- and outlets. The brachiocephalic, left common carotid, and left subclavian arteries (first, second and third branches in the arch) were extended $30 \mathrm{~mm}$ to minimize numerical problems at the outlets and care was taken to insure that no sharp corners were present where the vessels branched from the arch. No other minor vessels leaving the aorta were included. High quality hexahedral computational meshes were constructed in ANSYS ICEM CFD 13.0 (ANSYS Inc. Canonsburg, PA, USA). The mesh quality metric in ICEM has a range from -1 to 1 , where -1 is unacceptable, 1 is perfect, and 0.2 is usually considered as an acceptable threshold value. In total, $98.5 \%$ of the mesh elements had a quality above 0.75 and remaining elements had a quality above 0.35 . As one of the goals
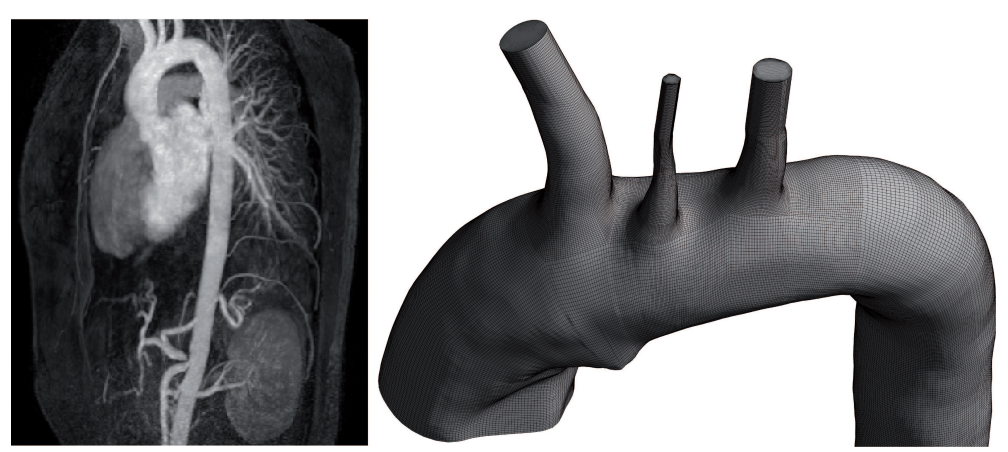

Fig. 1. Left: Maximum intensity projection MRI of the aorta used in the simulation. Right: Partial view of the aorta and the structured computational mesh. 
with the simulation was to resolve the WSS, special attention was taken to have a good resolution close to the wall. The boundary layer was captured with 30 prism layers adjacent to the wall where the first layer thickness was approximately $10 \mu \mathrm{m}$, and subsequent layers grew exponentially until the thickness of the last layer matched the core mesh size. A measure of near-wall resolution is the dimensionless wall distance $y^{+}$, and for highly accurate simulations where the flow in the boundary layer is important, a $y^{+}$on the order of $1-2$ is needed. In the simulations it had a maximum value of 1.25 and a mean value of 0.25 during a cardiac cycle, which provided a very good resolution close to the wall.

Mesh independency tests were carried out with 2.8 MC (million cells), 4.8 MC, and 12.1 MC. It was found that the $u, v$, and $w$ velocity profiles in the descending aorta were identical (within $5 \%$ ) for all three meshes. The 2.8 MC mesh differed in average WSS distribution compared to the larger meshes which were very similar, so it was decided to use the $4.8 \mathrm{MC}$ mesh in the computations.

\subsection{Fluid Model}

The fluid was set to resemble blood with a constant viscosity of 3.5e-3 Pa s (i.e. Newtonian fluid) and a density of $1080 \mathrm{~kg} / \mathrm{m}^{3}$. From the velocity profiles in the MRI measurements, the Reynolds number based on inlet diameter, ranged from 150 at late diastole to approximately 6000 at peak systole, with a mean of 1200. As velocity profiles were measured with MRI in a plane in both the ascending and descending aorta, a physiological inlet boundary condition could be set in the ascending aorta. Also, from the measured velocities, the flow leaving the arteries in the aortic arch could be deducted, as the difference between the ascending and descending flow rates. Mass flow rates were specified on each of the three arteries in the aortic arch; the difference between ascending and descending flow times a scale factor were used. The scale factors were based on cross-sectional area and were: 10/16, 1/16, 5/16, for the brachiocephalic, left common carotid, and left subclavian artery (1st, 2nd, and 3rd branch), respectively. In the descending aorta a time-dependent pressure was calculated using a three-element Windkessel model. The model uses the mass flow rate to compute a physiological pressure, and is 
plotted in Fig. 2, together with the ascending and descending mass flow rates. For further details on the Windkessel implementation, see [16].
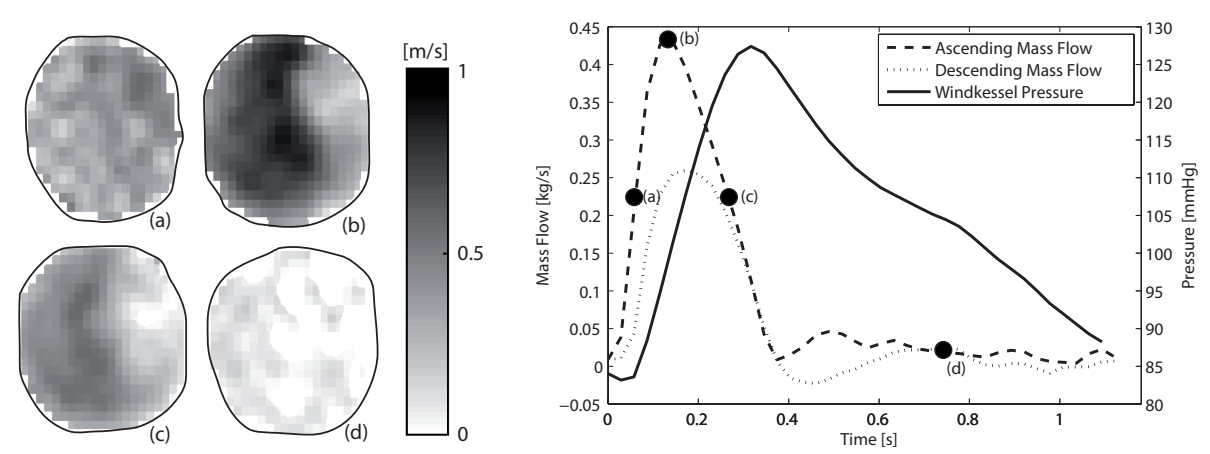

Fig. 2. Left: Example of measured flow profile in the ascending aorta at max acceleration (a), peak systole (b), mac deceleration (c), mid diastole (d). Right: The measured mass flow in the ascending and descending aorta, and the prescribed pressure at the outlet in the thoracic aorta.

\subsection{LES Modeling}

With the Reynolds number in the range of transitional flow, care must be taken to accurately compute the flow field. LES is a technique that separates between large and small scales in the flow, and the scales larger than a filter width (normally the grid spacing) are resolved while the smaller scales are modeled. The governing equations are obtained by filtering the time-dependent Navier-Stokes equations which then yields an extra term $-\frac{\partial \tau_{i j}}{\partial x_{j}}$, where $\tau_{i j}$ denotes the subgrid-scale stresses which includes the effect from the small scales. The subgrid-stresses are related to the large-scale strain rate tensor $\bar{S}_{i j}$ through the eddy-viscosity hypothesis:

$$
\tau_{i j}-\frac{1}{3} \delta_{i j} \tau_{k k}=2 v_{s g s} \bar{S}_{i j}
$$


where $v_{s g s}$ is the eddy viscosity and $\bar{S}_{i j}$ is the resolved strain rate defined as:

$$
\bar{S}_{i j}=\frac{1}{2}\left(\frac{\partial \bar{u}_{i}}{\partial x_{j}}+\frac{\partial \bar{u}_{j}}{\partial x_{i}}\right)
$$

Overbar on a variable (e.g. $\bar{u}_{i}$ ) means that it is filtered. The subgrid-scale eddy viscosity was modeled with the wall-adapted local eddy-viscosity (WALE) LES model by [17]. It was designed to eliminate the subgrid contribution in shear flows and to return the correct near-wall scaling of the subgrid eddyviscosity $\left(v_{t}=O\left(y^{3}\right)\right)$ without any damping functions. Nicoud and Ducros [17] also showed that the model can handle transition regimes. The eddy-viscosity in the WALE model is defined as:

$$
v_{s g s}=\left(C_{w a l e} \Delta\right)^{2} \frac{\left(S_{i j}^{d} S_{i j}^{d}\right)^{3 / 2}}{\left(\bar{S}_{i j} \bar{S}_{i j}\right)^{5 / 2}+\left(S_{i j}^{d} S_{i j}^{d}\right)^{5 / 4}}
$$

where $C_{\text {wale }}$ is a constant set to 0.5 based on results from homogeneous isotropic turbulence, $\Delta$ is the cube root of the computational cell volume, and $S_{i j}^{d}$ is the traceless symmetric part of the square of the velocity gradient tensor. The $S_{i j}^{d}$ tensor can be rewritten in terms of (filtered) strain-rate and vorticity tensors as:

$$
S_{i j}^{d}=\bar{S}_{i k} \bar{S}_{k j}+\bar{\Omega}_{i k} \bar{\Omega}_{k j}-\frac{1}{3} \delta_{i j}\left(\bar{S}_{m n} \bar{S}_{m n}-\bar{\Omega}_{m n} \bar{\Omega}_{m n}\right)
$$

where the strain-rate is defined in Eqn. 2 and the vorticity tensor is:

$$
\bar{\Omega}_{i j}=\frac{1}{2}\left(\frac{\partial \bar{u}_{i}}{\partial \bar{x}_{j}}-\frac{\partial \bar{u}_{j}}{\partial \bar{x}_{i}}\right) .
$$

The simulations were performed with ANSYS CFX 13.0 (ANSYS Inc. Canonsburg, PA, USA). A maximum root-mean-square (RMS) residual of $10^{-6}$ was defined for the momentum and continuity equations, and domain imbalances were converged well below $0.05 \%$. Independence tests were carried out on the convergence criteria to ensure an accurate solution. Temporal discretization was performed 
with a second order backward Euler scheme and the spatial discretization used second order central differencing. The time step was 1e-4 s yielding an average Courant number of approximately 0.4. To eliminate initial transient effects, 5 cardiac cycles were run before saving of data began. The simulations were run on the Linux clusters Neolith and Kappa at National Supercomputer Centre (NSC), Linköping, Sweden.

\subsection{Post Processing}

Consecutive cardiac wave forms in vivo may be slightly different to each other as the heart is not a perfectly reproducible pump and local flow disturbances may be present. The flow measurements were taken when the heart rate was 53 beats per minute, which was set as a constant heart rate in the model. However, flow disturbances will occur, predominately during the latter part of systole due to the high peak Reynolds number $(\approx 6000)$, making consecutive pulses slightly different as indicated by the WSS signals seen in Fig. 3. The figure demonstrates the need of a statistical representation, where the average flow quantities over a large number of pulses are considered.

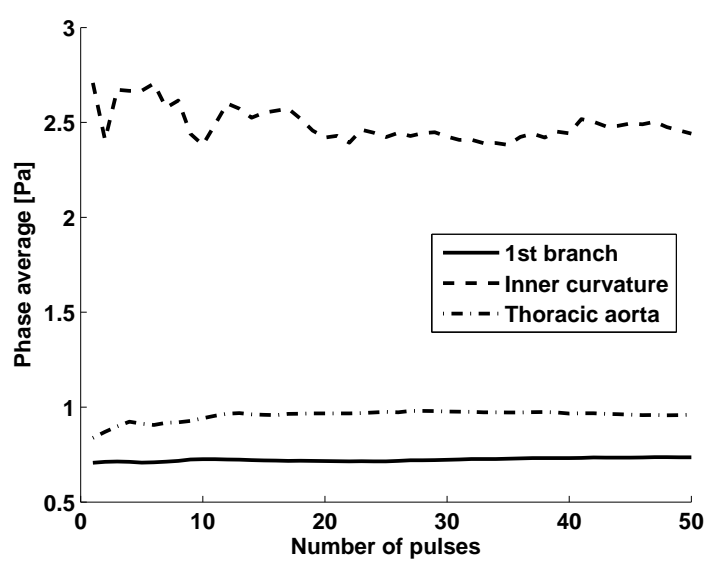

Fig. 3. A representative example of the WSS magnitude on the inner curve of the aortic arch during three consecutive cardiac cycles. The disturbed flow causes the WSS to be somewhat different for each cycle, especially during the deceleration phase.

A flow variable $\phi$ in a periodic pulsatile flow can be decomposed to a phase average and a fluctuating 
component, as:

$$
\phi(\mathbf{x}, t)=\langle\phi\rangle(\mathbf{x}, t)+\phi^{\prime}(\mathbf{x}, t)
$$

The phase average operator $\langle$.$\rangle is defined as:$

$$
\langle\phi\rangle(\mathbf{x}, t)=\frac{1}{N} \sum_{n=0}^{N-1} \phi(\mathbf{x}, t+n T)
$$

where $\mathrm{N}$ is the number of cardiac cycles and $\mathrm{T}$ the (constant) period of the cardiac cycle. The phaseaverage is the proper statistical representation of a pulsating flow where disturbances are present. The fluctuating part, denoted with a prime, was computed as a root mean square (RMS) of the instantaneous signal and the phase average:

$$
\phi^{\prime}(\mathbf{x}, t)=\sqrt{\frac{1}{N} \sum_{n=0}^{N-1}(\phi(\mathbf{x}, t+n T)-\langle\phi\rangle(\mathbf{x}, t))^{2}}
$$

This decomposition was applied to WSS, yielding the notation:

$$
\mathrm{WSS}=<\mathrm{WSS}>+\mathrm{WSS}^{\prime}
$$

for the phase-average and fluctuating part, respectively. The time-averaged WSS (TAWSS) was computed as:

$$
<\mathrm{TAWSS}>=\frac{1}{T} \int_{0}^{T}<\mathrm{WSS}>(\mathbf{x}, t) d t
$$

where the notation $<$ TAWSS $>$ notation is used to emphasize that the time-averaged WSS is based on phase-averaged values and not a single cardiac cycle. In a periodic flow where each cycle can be seen as independent of each other, the phase average effectively filters out background turbulence while the time average removes both background turbulence and periodic effects [18]. 
The WSS vector components at each mesh vertex on the computational grid were exported every $0.01 \mathrm{~s}$ during 50 cardiac cycles, yielding close to two Billion data points used in the post-processing. The number of cardiac cycles needed to reach statistical convergence was investigated by calculating the phase average using Eqn. 7 for $n=1 . .50$ number of pulses and plotting the result. When the curves levelled out, statistical convergence was decided to be found. In Fig. 4 are three curves plotted, displaying the phase average of representative points on the aortic surface at a time in the deceleration phase when most disturbances were found. It is seen that the phase average changes during the first 15 pulses but then becomes essentially flat with very small changes $( \pm 0.02 \mathrm{~Pa})$. For other times in the cardiac cycle the curves levelled out faster due to smaller amount of disturbances. It was therefore decided that 50 cardiac cycles were enough for reliable statistics.

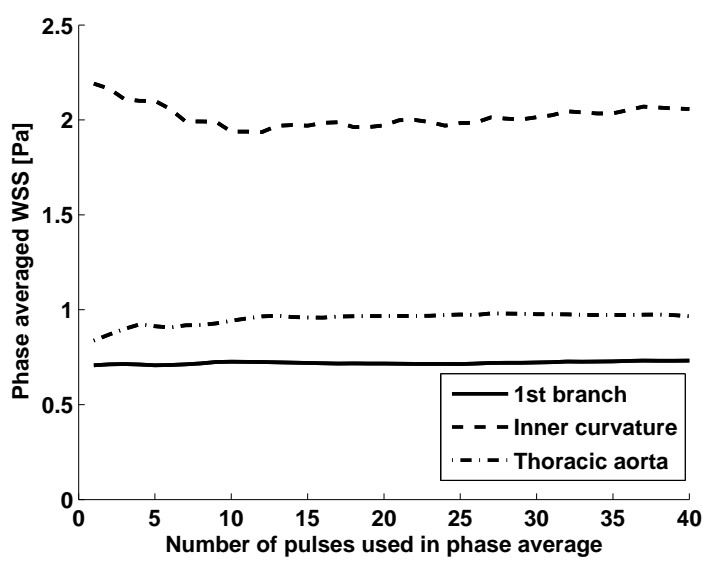

Fig. 4. Statistical convergence for three points on the arterial surface. The curves are phase-averages computed on 1 to 50 cardiac cycles and are located close to the first branch, on the inner curvature of the aorta and in the thoracic aorta. The values are at a time in the deceleration phase, when most disturbances are present.

A parameter often used in literature to quantify unsteady WSS is the Oscillatory Shear Index (OSI), which describes the cyclic departure of the WSS vector from its predominant axial alignment $[2,19]$. It 
is defined (using phase-average values) as:

$$
<\mathrm{OSI}>=0.5\left(1-\frac{\left|\int_{0}^{T}<\mathrm{WSS}>d t\right|}{\int_{0}^{T}|<\mathrm{WSS}>| d t}\right)
$$

The values for $<$ OSI $>$ ranges from 0 to 0.5 , were zero means that the instantaneous WSS vector is aligned with the time-averaged vector throughout the entire pulse and, thus, do not oscillate at all. On the other hand, 0.5 means that the instantaneous vector never is aligned with the time-averaged vector, which indicates a very oscillatory behavior.

\section{Results and Discussion}

\subsection{Aortic Blood Flow}

To understand the flow dynamics in the aorta, velocity contours and vectors in the aortic arch and descending aorta are plotted in Figs. 5 and 6 at four time points during systole. At max acceleration the flow in the arch is asymmetric due to the skew velocity profile in the ascending aorta and also due to the flow leaving through the branches. In the descending aorta, however, the flow quickly becomes well defined with a flat velocity profile. This is clearly seen in Fig 6, which shows a uniform flow field in the descending aorta. On the outer curvature after the third branch there is a region where the flow is divided into flow going back into the branch and flow going down in the descending aorta. This region is likely to exhibit both oscillating WSS magnitude and direction during a cardiac cycle, due to the pulsatile nature of the flow. A large scale helical flow structure forms in the ascending aorta and moves through the arch. The rotation is clockwise when viewed in the direction of forward movement, similar to in vivo measurements by [20,21] and simulation results by e.g. [22].

As the velocity increases during peak systole, a small jet of accelerated flow emerges close to the inner curvature of the arch, creating a separation zone. As the fluid in the separation zone is almost stationary, while the velocity of the flow in the jet is high, large velocity gradients are present in that 
region. Overall, the flow field is still well structured in the descending and thoracic aorta, but in the arch the asymmetric flow prevails.

During the deceleration phase the flow breaks up into a disturbed, turbulent flow field. This is represented by very complicated flow contours and large streaks where vectors point in all directions. The separation zone on the inner curvature is still present and a second separation zone has formed a small distance downstream. At the end of systole a completely disturbed flow field is formed, with local flow reversal in the entire aorta. The velocity is low, and there is no preferred flow direction. The helical structure found at the acceleration phase and peak systole is dissolved and replaced by small turbulent flow structures. This unstructured flow behavior is present throughout diastole, until the next cardiac cycle starts and the flow acceleration recreates the well defined flow situation again.

The mass flow to the branches decreases in the deceleration phase and is essentially zero during diastole, indicating that the main flow contribution is during the acceleration phase and peak systole.

\subsection{Integrated WSS Variables}

The time-averaged WSS is normally reported in literature as it is the shear load over time that is subjected to the arterial wall, while OSI describes the changes of WSS over a cardiac cycle. A drawback is that $<$ OSI $>$ does not account for the WSS magnitude, and to overcome this, it is here used together with $<$ TAWSS $>$. It has been found that both WSS and OSI are important with respect to formation and stability of atherosclerotic plaques (see e.g. [23]), and that they can be used to determine the complexity of lesions $[24,25]$. The values for $<\mathrm{OSI}>$ and $<$ TAWSS $>$ were computed for the entire aortic arch and plotted to the left in Fig. 7. In total, approximately 113000 points were computed, where each point represents a mesh vertex on the aortic surface mesh. The graph clearly shows that low time-averaged WSS is dominant when OSI take on values from zero to 0.5 . An inverse relation between $<$ OSI $>$ and $<$ TAWSS $>$ is also seen, with low values of $<$ OSI $>$ corresponding to high $<$ TAWSS $>$ which is in line with findings from other studies e.g. [26,27]. As the oscillating shear variable is insensitive to 

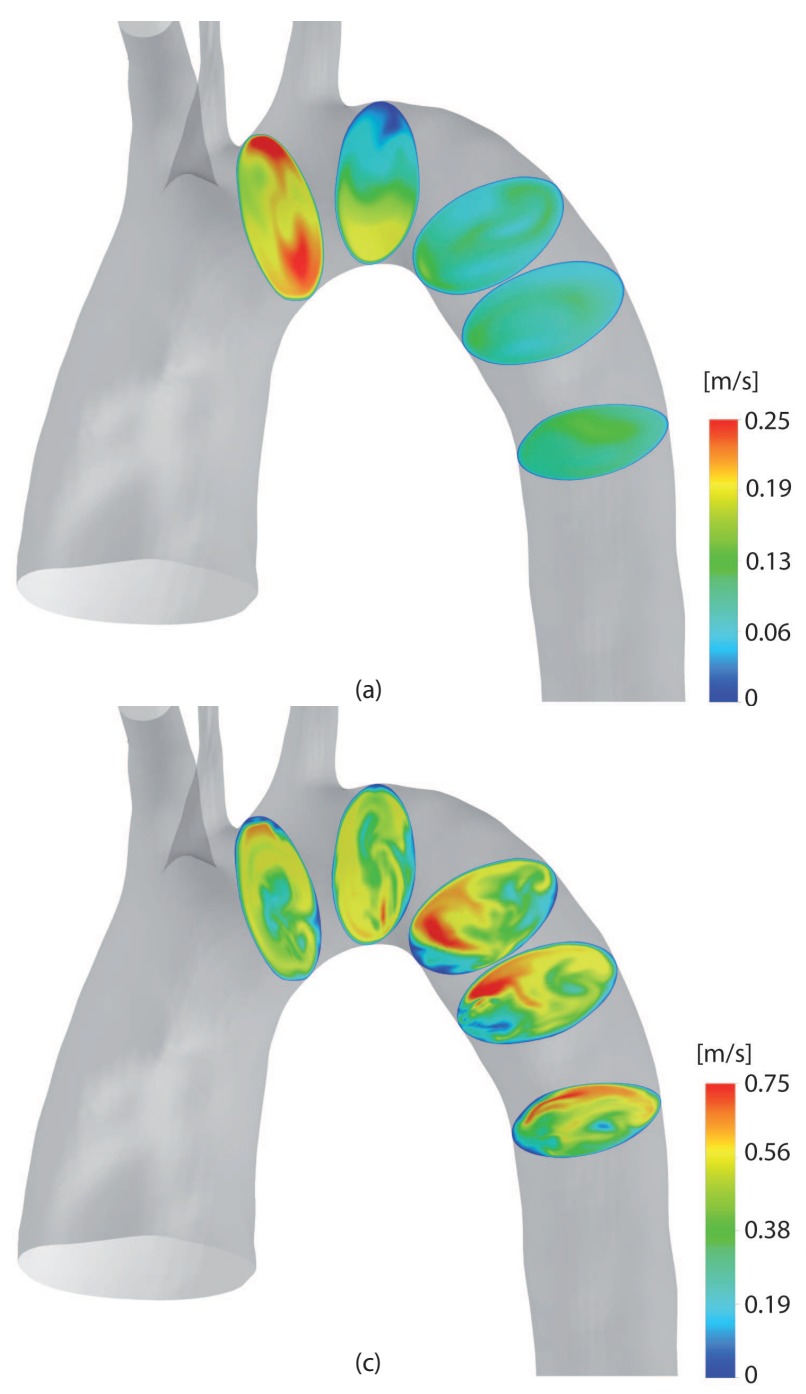

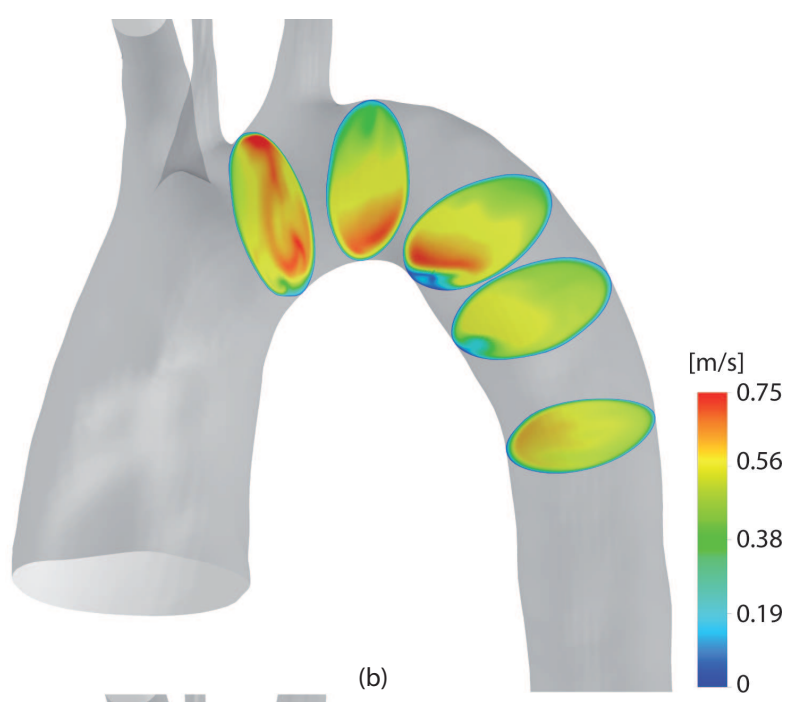

(b)

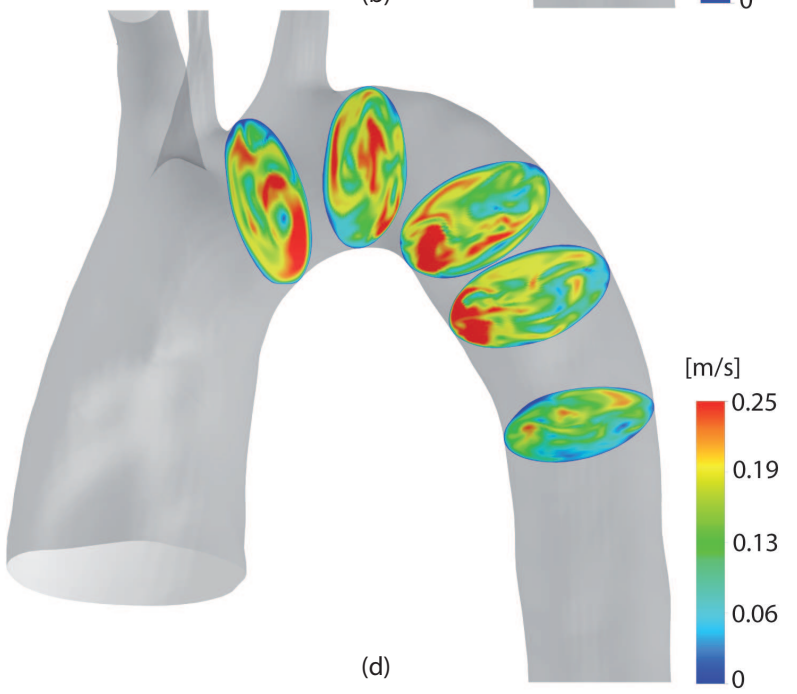

Fig. 5. Flow contours in the aortic arch and descending aorta during systole at max acceleration (a), peak systole (b), max deceleration (c), and end systole (d). Notice the different color scale.

WSS magnitude, and the WSS vector is pointing in random directions with very low magnitudes during diastole, most of the elevated $<$ OSI $>$ values are are present at low time-averaged WSS values.

However, there were, interestingly a few points that departed from the inverse relationship and exhibited elevated values for both parameters, indicated by the dotted box. The locations of these points were mapped back onto the aortic surface and is displayed in the figure to the right. As clearly seen, these regions are only localized in the immediate vicinity of the branches and partly on the inner curvature of the arch, which are locations prone to atherosclerotic development [1-3]. Notice that there are only 

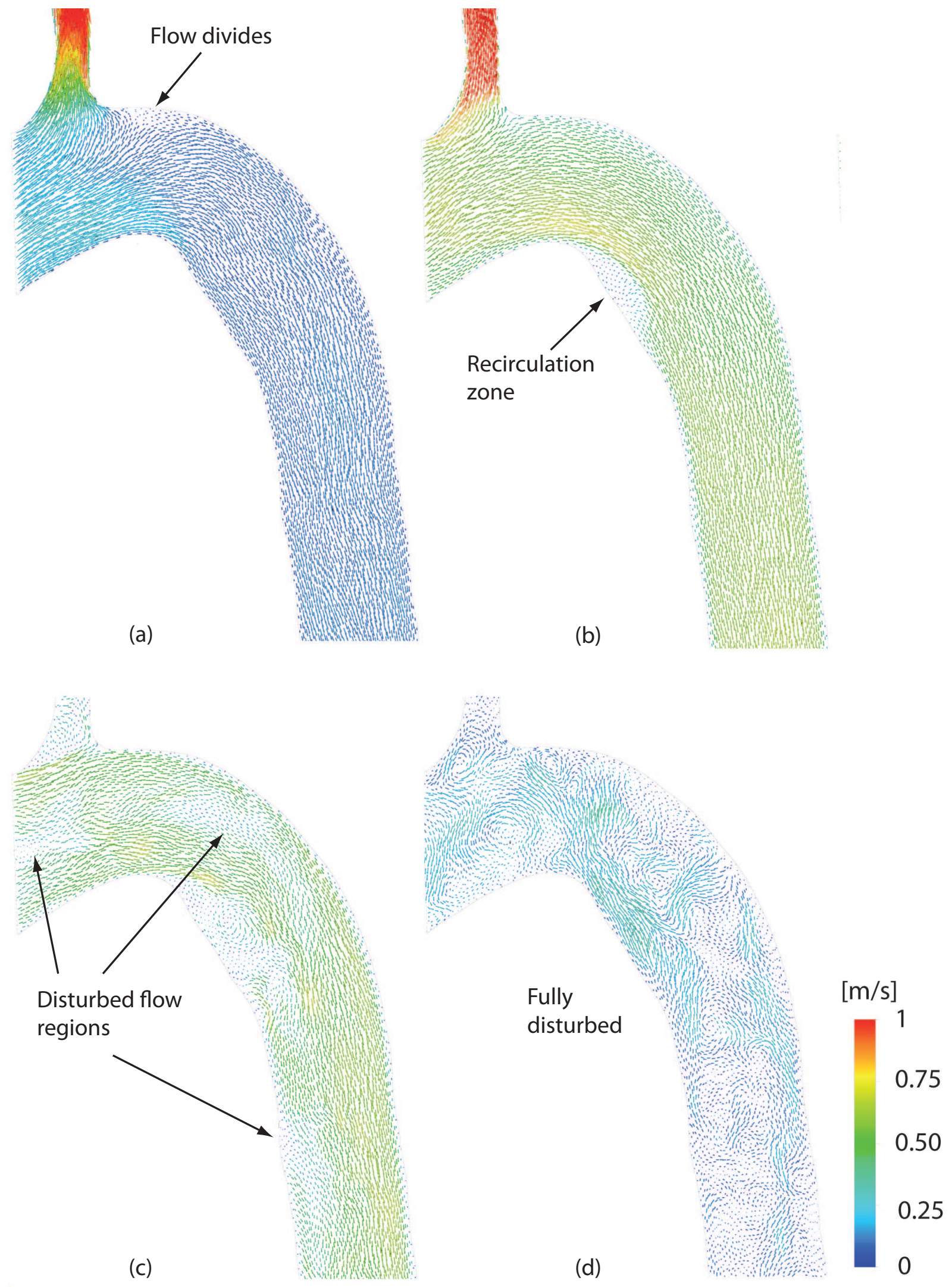

Fig. 6. Flow vectors in the aortic arch and descending aorta during systole at max acceleration (a), peak systole (b), max deceleration (c), and end systole (d). 
$<$ TAWSS $>$ values larger than 2 Pa that are present within these regions, which must be considered high compared to the rest of the aorta, especially since these locations are also oscillatory.
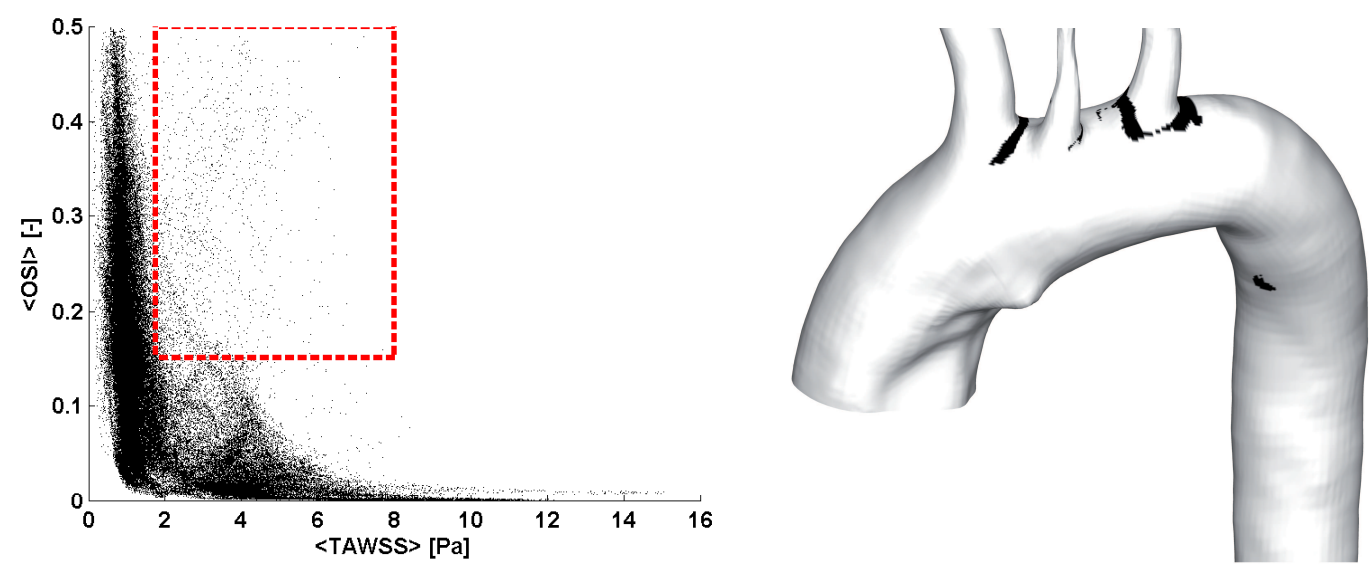

Fig. 7. To the left $\langle\mathrm{OSI}\rangle$ is plotted against $\langle$ TAWSS $\rangle$, using 113000 points on the aortic surface. The points inside the dotted box were determined to be elevated values of both parameters, and were mapped back onto the aorta (dark spots in right figure). It was found that these locations were located in the vicinity of the branches in the aortic arch and at a small location in the recirculation region on the inner curvature of the descending aorta.

\subsection{Spatial and Temporal WSS Variation}

To further investigate the WSS in the descending aorta, the WSS signal was first decomposed using Eqn. 9 to a phase-average and a fluctuating part, and the time average, Eqn. 10 was also computed for reference. Eight points on the surface were investigated, named P1-P8. The points were located around the branches, on the inner and outer curvature on the descending aorta, and in the thoracic aorta, see Fig. 8. Regarding the two points located close to the first branch in the arch, there is a large difference in magnitude upstream and downstream the branch. The first point, P1, is located on the beginning of the branch but in a region where the geometry of the arch gradually changes into the branching vessel like a funnel, while the second point, $\mathrm{P} 2$, is located on the opposite side of the vessel. This small difference in location is represented by a huge difference in WSS magnitude: P2 is approximately six times larger 


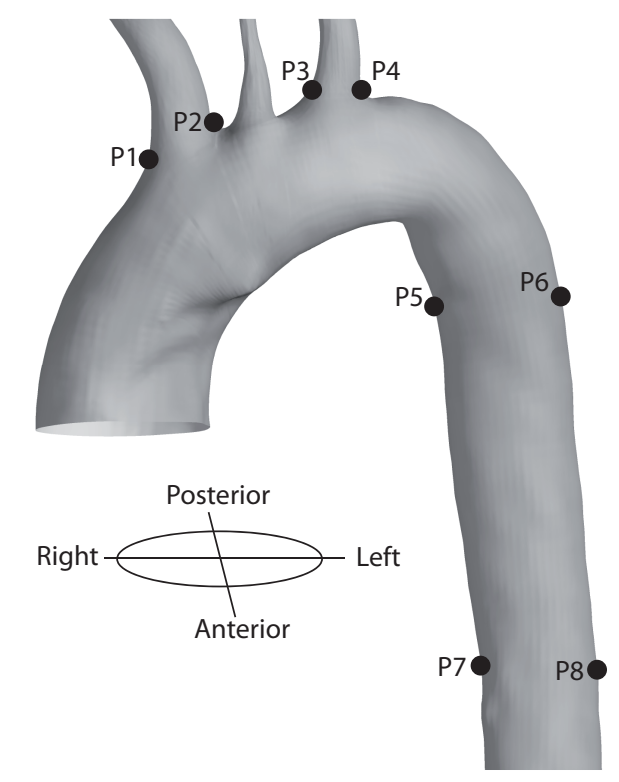

Fig. 8. Location of points used for WSS decomposition.

than $\mathrm{P} 1$ and the fluctuating $\mathrm{WSS}^{\prime}$ is less significant for $\mathrm{P} 1$ compared to $\mathrm{P} 2$. The fluctuating component $\mathrm{WSS}^{\prime}$, is very large for P2 during systole, which shows that not only is the WSS magnitude high, but there are also significant fluctuations present in systole. During the acceleration phase, it is even larger than $<$ TAWSS $>$, indicating large turbulent fluctuations of the WSS vector. The results from the $<$ OSI $>$ calculation also suggests that this is a location where large oscillating WSS magnitudes are present, as the location for P2 coincides with one of the dark spots in Fig. 7. Also notice how the WSS magnitude oscillates for both points during diastole (0.35-1.1 s), which again indicates the complex flow situation around the branches.

Points 3 and 4, which are located on the third branch are qualitatively similar to each other, with high $<$ WSS $>$ values during systole and small oscillating parts during diastole. The $\mathrm{WSS}^{\prime}$ is most significant during the acceleration phase with values around $5 \mathrm{~Pa}$, which is larger than the $<$ TAWSS $>$ on most places on the aorta. It decreases fast after in the late part of systole and becomes insignificant in diastole. It can also be seen here that the large amount of fluctuations and high $<$ TAWSS $>$ in P3 and P4 results in high $<$ OSI $>$ values and, hence, correlates with the dark parts in Fig. 7. 
The WSS signals in the inner and outer curvature of the descending aorta is represented by points P5 and P6. The maximum $<\mathrm{WSS}>$ is equal, but while point P6 shows a gradual increase of $<\mathrm{WSS}>$ during the acceleration phase and a rapid decrease during deceleration and almost no contribution of WSS ${ }^{\prime}$, point P5 shows the opposite: fluctuating $<\mathrm{WSS}>$ values through the entire systolic phase and considerable fluctuations. This is caused by the formation of a recirculation zone on the inner curvature which considerably alters the WSS signal. In this region the flow is very disturbed with significant differences between consecutive cardiac cycles. The fluctuations caused by the turbulent flow are manifested by a large $\mathrm{WSS}^{\prime}$, which is even larger than $<$ TAWSS $>$ during an extended time in systole.

In the thoracic aorta the flow situation is much less disturbed which is reflected by the fact that the WSS in points P7 and P8 are qualitatively similar with a well defined increase and decrease of $<$ WSS $>$ following the mass flow pulse. The $\mathrm{WSS}^{\prime}$ is low throughout the pulse which is another sign of an undisturbed flow.
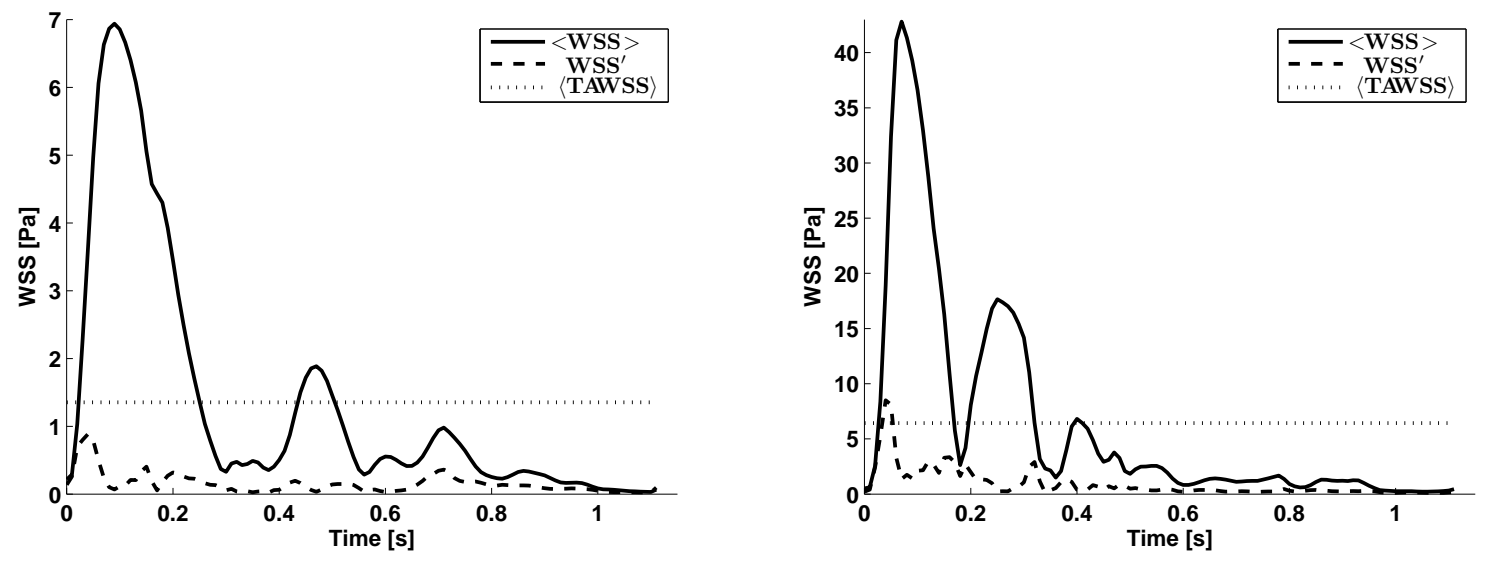

Fig. 9. Distribution of $\left\langle\right.$ WSS $>$, WSS ${ }^{\prime}$ and $\langle$ TAWSS $>$ in points $\mathrm{P} 1$ and $\mathrm{P} 2$, located upstream and downstream the first branch. Notice the different scales on the $y$-axis. 

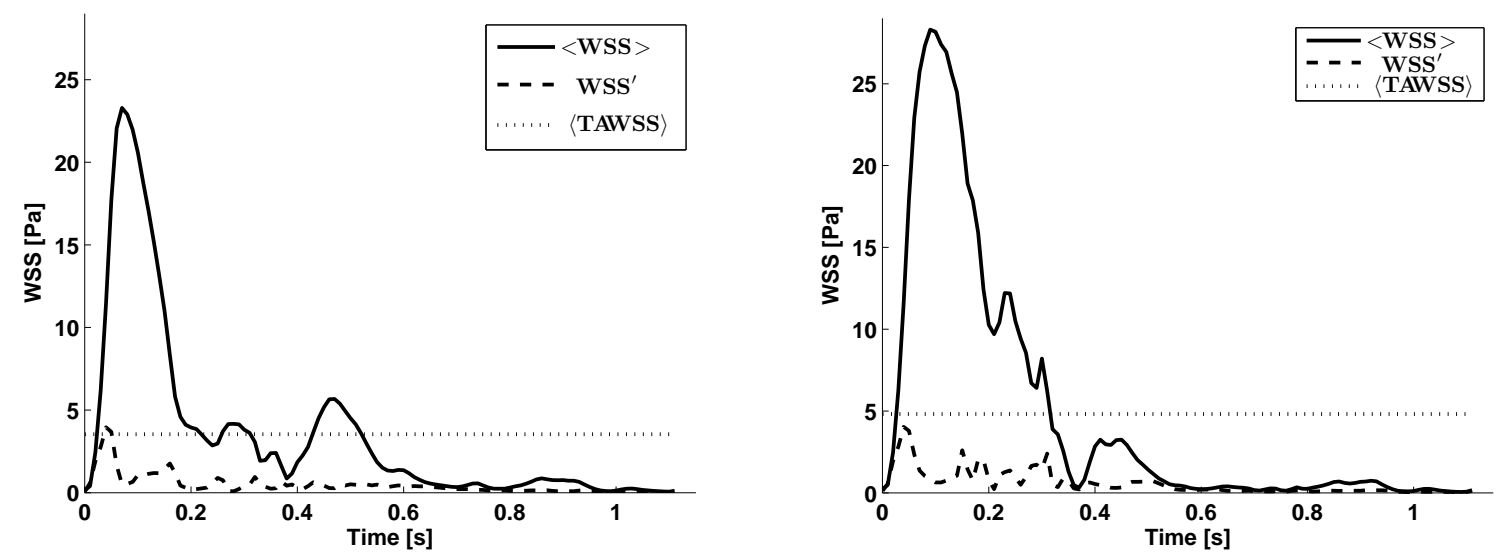

Fig. 10. Distribution of $\left\langle\right.$ WSS $>$, WSS ${ }^{\prime}$ and $\langle$ TAWSS $>$ in points $\mathrm{P} 3$ and $\mathrm{P} 4$, located upstream and downstream the third branch.
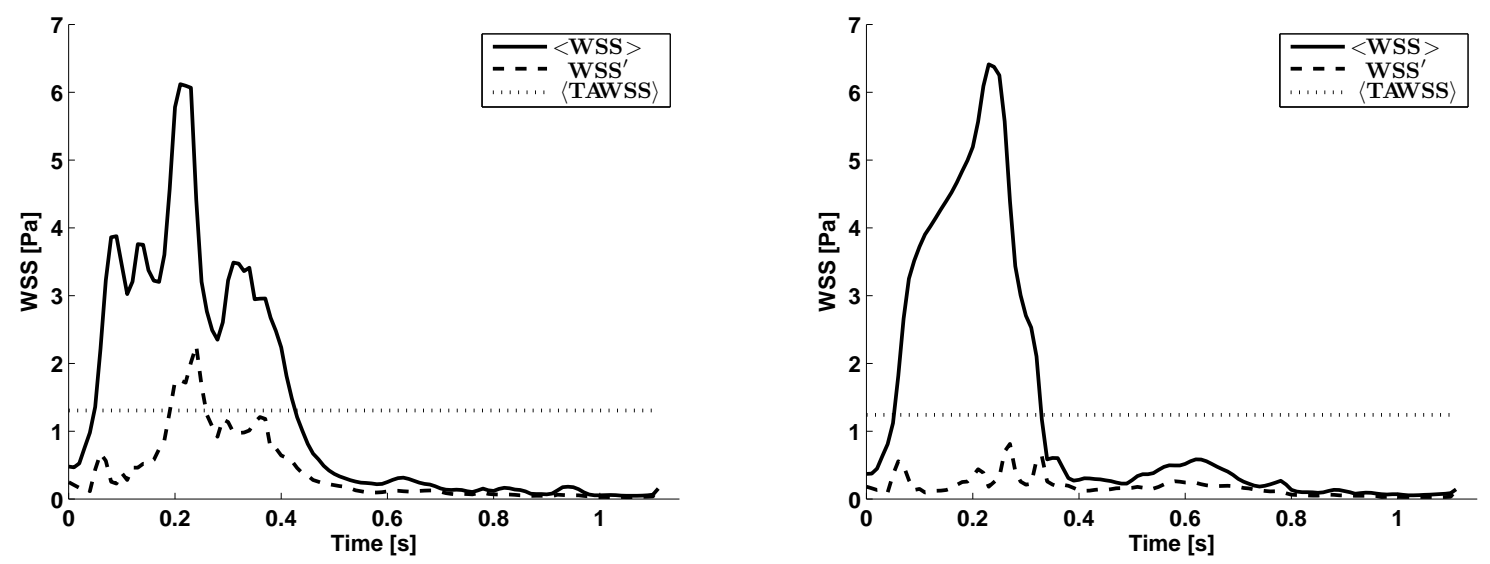

Fig. 11. Distribution of $\left\langle\right.$ WSS $>$, WSS ${ }^{\prime}$ and $\langle$ TAWSS $>$ in points P5 and P6, located on the inner and outer curvature in the descending aorta.
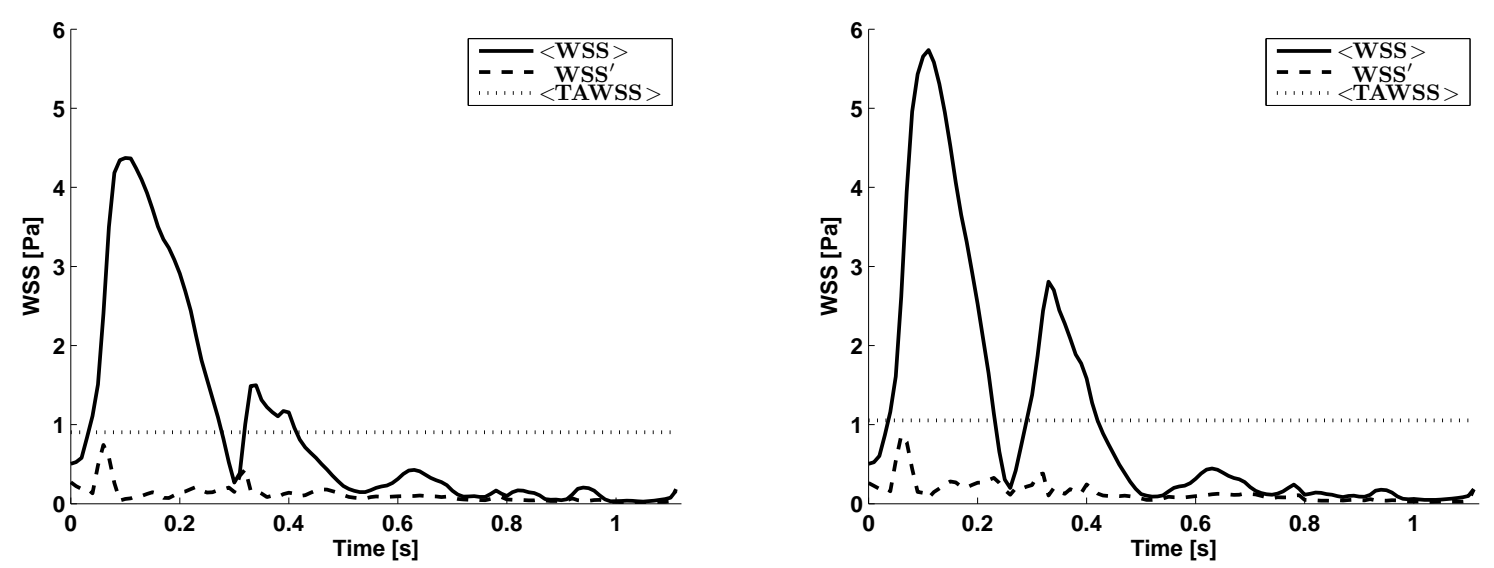

Fig. 12. Distribution of $\left\langle\right.$ WSS $>$, WSS ${ }^{\prime}$ and $\langle$ TAWSS $>$ in points $\mathrm{P} 7$ and P8, located on the right and left side on the thoracic aorta. 


\section{Concluding Remarks}

In this study we investigated the WSS in a subject specific human aorta using a LES turbulence model and measured velocity profiles as boundary conditions, to increase the physiological relevance. The complex flow situation in the aorta was shown in detail and the WSS was investigated using both integrated and instantaneous values. The use of a scale resolving turbulence model allows computation of fluctuating flow variables, such as WSS' which was introduced as a decomposition similar to Reynolds decomposition.

To conclude, this decomposition of WSS into a pulsating and a fluctuating part increases the understanding of how WSS affects the aortic wall, and allows for both qualitative and quantitative comparisons to be made. Future work includes the application of the present methodology to a larger cohort of subjects.

\section{Acknowledgments}

This work was supported by grants from the Swedish research council, VR 2007-4085 and VR 20104282. The Swedish National Infrastructure for Computing (SNIC) is acknowledged for computational resources provided by the National Supercomputer Centre (NSC) under grant No. SNIC022/09-11. Dr. Tino Ebbers at the Department of Medicine and Care at Linköping University is acknowledged for the MRI measurements. This work has been conducted in collaboration with the Center for Medical Image Science and Visualization (CMIV, http://www.cmiv.liu.se/) at Linköping University, Sweden. CMIV is acknowledged for provision of financial support and access to leading edge research infrastructure. 


\section{References}

[1] J. J. Chiu, S. Usami, and S. Chien. Vascular endothelial responses to altered shear stress: pathologic implications for atherosclerosis. Ann. Med., 41:19-28, 2009.

[2] D. N. Ku, D. P. Giddens, C. K. Zarins, and S. Glagov. Pulsatile flow and atherosclerosis in the human carotid bifurcation. Positive correlation between plaque location and low oscillating shear stress. Arteriosclerosis, 5:293-302, 1985.

[3] A. G. Passerini, A. Milsted, and S. E. Rittgers. Shear stress magnitude and directionality modulate growth factor gene expression in preconditioned vascular endothelial cells. J. Vasc. Surg., 37:182190, Jan 2003.

[4] S. A. Ahmed and D. P. Giddens. Velocity measurements in steady flow through axisymmetric stenoses at moderate Reynolds numbers. J Biomech, 16:505-516, 1983.

[5] S. A. Ahmed and D. P. Giddens. Flow disturbance measurements through a constricted tube at moderate Reynolds numbers. J Biomech, 16:955-963, 1983.

[6] S. A. Ahmed and D. P. Giddens. Pulsatile poststenotic flow studies with laser Doppler anemometry. J Biomech, 17:695-705, 1984.

[7] S. S. Varghese and S. H. Frankel. Numerical modeling of pulsatile turbulent flow in stenotic vessels. J Biomech Eng, 125:445-460, Aug 2003.

[8] J. Ryval, A. G. Straatman, and D. A. Steinman. Two-equation turbulence modeling of pulsatile flow in a stenosed tube. J Biomech Eng, 126:625-635, Oct 2004.

[9] J. Banks and N. W. Bressloff. Turbulence modeling in three-dimensional stenosed arterial bifurcations. J Biomech Eng, 129:40-50, Feb 2007.

[10] F. P. Tan, G. Soloperto, S. Bashford, N. B. Wood, S. Thom, A. Hughes, and X. Y. Xu. Analysis of flow disturbance in a stenosed carotid artery bifurcation using two-equation transitional and turbulence models. J Biomech Eng, 130:061008, Dec 2008. 
[11] S. S. Varghese, S. H. Frankel, and P. F. Fischer. Modeling transition to turbulence in eccentric stenotic flows. J Biomech Eng, 130:014503, Feb 2008.

[12] F. P. Tan, N. B. Wood, G. Tabor, and X. Y. Xu. Comparison of LES of Steady Transitional Flow in an Idealized Stenosed Axisymmetric Artery Model With a RANS Transitional Model. J Biomech Eng, 133:051001, May 2011.

[13] S. S. Varghese, S. H. Frankel, and P. F. Fischer. Direct numerical simulation of stenotic flows. Part 1. Steady flow. Journal of Fluid Mechanics, 582:253-280, 2007.

[14] R. Gårdhagen, J. Lantz, F. Carlsson, and M. Karlsson. Quantifying turbulent wall shear stress in a stenosed pipe using large eddy simulation. J Biomech Eng, 132:061002, Jun 2010.

[15] E. Heiberg, J. Sjögren, M. Ugander, M. Carlsson, H. Engblom, and H. Arheden. Design and validation of Segment-freely available software for cardiovascular image analysis. $B M C M e d$ Imaging, 10:1, 2010.

[16] J. Lantz, J. Renner, and M. Karlsson. Wall Shear Stress in a Subject Specific Human Aorta Influence of Fluid-Structure Interaction . Submitted, 2011.

[17] F. Nicoud and F. Ducros. Subgrid-scale stress modelling based on the square of the velocity gradient tensor. Flow, Turbulence and Combustion, 62:183-200, 1999.

[18] G.J. Brereton and W.C Reynolds. Dynamic response of boundary-layer turbulence to oscillatory shear. Physics of Fluids A:Fluid Dynamics, 3(1):178-187, Jan 1991.

[19] X. He and D. N. Ku. Pulsatile flow in the human left coronary artery bifurcation: average conditions. J Biomech Eng, 118:74-82, Feb 1996.

[20] P. J. Kilner, G. Z. Yang, R. H. Mohiaddin, D. N. Firmin, and D. B. Longmore. Helical and retrograde secondary flow patterns in the aortic arch studied by three-directional magnetic resonance velocity mapping. Circulation, 88:2235-2247, Nov 1993.

[21] J. P. Kvitting, T. Ebbers, L. Wigstrom, J. Engvall, C. L. Olin, and A. F. Bolger. Flow patterns in 
the aortic root and the aorta studied with time-resolved, 3-dimensional, phase-contrast magnetic resonance imaging: implications for aortic valve-sparing surgery. J. Thorac. Cardiovasc. Surg., 127:1602-1607, Jun 2004.

[22] M. Nakamura, S. Wada, and T. Yamaguchi. Computational analysis of blood flow in an integrated model of the left ventricle and the aorta. J Biomech Eng, 128:837-843, Dec 2006.

[23] C. Cheng, D. Tempel, R. van Haperen, A. van der Baan, F. Grosveld, M. J. Daemen, R. Krams, and R. de Crom. Atherosclerotic lesion size and vulnerability are determined by patterns of fluid shear stress. Circulation, 113:2744-2753, Jun 2006.

[24] A. Frydrychowicz, A. F. Stalder, M. F. Russe, J. Bock, S. Bauer, A. Harloff, A. Berger, M. Langer, J. Hennig, and M. Markl. Three-dimensional analysis of segmental wall shear stress in the aorta by flow-sensitive four-dimensional-MRI. J Magn Reson Imaging, 30:77-84, Jul 2009.

[25] C. Irace, C. Cortese, E. Fiaschi, C. Carallo, E. Farinaro, and A. Gnasso. Wall shear stress is associated with intima-media thickness and carotid atherosclerosis in subjects at low coronary heart disease risk. Stroke, 35:464-468, Feb 2004.

[26] B. T. Tang, T. A. Fonte, F. P. Chan, P. S. Tsao, J. A. Feinstein, and C. A. Taylor. Three-dimensional hemodynamics in the human pulmonary arteries under resting and exercise conditions. Ann Biomed Eng, 39:347-358, Jan 2011.

[27] X. Liu, Y. Fan, X. Deng, and F. Zhan. Effect of non-Newtonian and pulsatile blood flow on mass transport in the human aorta. J Biomech, 44:1123-1131, Apr 2011. 


\section{List of Figures}

1 Left: Maximum intensity projection MRI of the aorta used in the simulation. Right: Partial view of the aorta and the structured computational mesh. . . . . . . . . . 5

2 Left: Example of measured flow profile in the ascending aorta at max acceleration (a), peak systole (b), mac deceleration (c), mid diastole (d). Right: The measured mass flow in the ascending and descending aorta, and the prescribed pressure at the outlet in the

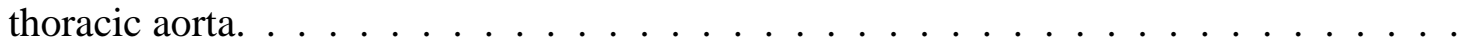

3 A representative example of the WSS magnitude on the inner curve of the aortic arch during three consecutive cardiac cycles. The disturbed flow causes the WSS to be somewhat different for each cycle, especially during the deceleration phase. . . . . . . . .

4 Statistical convergence for three points on the arterial surface. The curves are phaseaverages computed on 1 to 50 cardiac cycles and are located close to the first branch, on the inner curvature of the aorta and in the thoracic aorta. The values are at a time in the deceleration phase, when most disturbances are present. . . . . . . . . . . . . . .

5 Flow contours in the aortic arch and descending aorta during systole at max acceleration (a), peak systole (b), max deceleration (c), and end systole (d). Notice the different color scale.

6 Flow vectors in the aortic arch and descending aorta during systole at max acceleration (a), peak systole (b), max deceleration (c), and end systole (d) . . . . . . . . . . . 15 
7 To the left $<$ OSI $>$ is plotted against $<$ TAWSS $>$, using 113000 points on the aortic surface. The points inside the dotted box were determined to be elevated values of both parameters, and were mapped back onto the aorta (dark spots in right figure). It was found that these locations were located in the vicinity of the branches in the aortic arch and at a small location in the recirculation region on the inner curvature of the

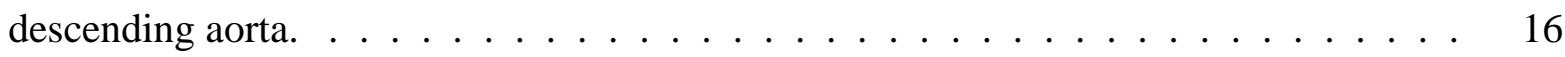

8 Location of points used for WSS decomposition. . . . . . . . . . . . . 17

9 Distribution of $<$ WSS $>$, WSS ${ }^{\prime}$ and $<$ TAWSS $>$ in points P1 and P2, located upstream and downstream the first branch. Notice the different scales on the y-axis. . . . . . . 18

10 Distribution of $<$ WSS $>$, WSS $^{\prime}$ and $<$ TAWSS $>$ in points P3 and P4, located upstream and downstream the third branch. . . . . . . . . . . . . . . . . . 19

11 Distribution of $<\mathrm{WSS}>, \mathrm{WSS}^{\prime}$ and $<$ TAWSS $>$ in points P5 and P6, located on the inner and outer curvature in the descending aorta. . . . . . . . . . . . . . . . . 19

12 Distribution of $<\mathrm{WSS}>, \mathrm{WSS}^{\prime}$ and $<$ TAWSS $>$ in points $\mathrm{P} 7$ and P8, located on the right and left side on the thoracic aorta. $\ldots \ldots \ldots \ldots \ldots$ 